\title{
Characterization of thin films using heavy ion beams
}

\author{
H TIMMERS*, R G ELLIMAN ${ }^{\dagger}$ and T R OPHEL ${ }^{\ddagger}$ \\ Department of Physics, University of Newcastle, Callaghan, NSW 2308, Australia \\ ${ }^{\dagger}$ Department of Electronic Materials Engineering, ${ }^{\ddagger}$ Department of Nuclear Physics, Research School of Physical \\ Sciences and Engineering, Australian National University, ACT 0200, Australia
}

\begin{abstract}
Thin films are used in many technological applications. The characterization of thin films requires compositional information as a function of sample depth. Ion beam analysis techniques, such as Rutherford backscattering spectrometry and elastic recoil detection (ERD), can provide this information in a uniform way for all elements and as absolute concentrations without relying on standards. These techniques can fully be exploited when projectile beams of heavy ions such as Si or Au are used. This improves the elemental resolution and the depth resolution when compared with standard He ion beam analysis. The use of gas ionization detectors increases detection efficiency and minimizes the beam exposure of the samples, so that the analysis is essentially nondestructive. The sampling depth of a few micrometers makes these techniques ideal for the stoichiometric analysis of the surface region of homogeneous materials and, in particular, thin surface films.
\end{abstract}

Keywords. Ion beam analysis; thin films; elastic recoil detection.

\section{Introduction}

Thin film materials are being developed for their use in many technological applications. The films need to be characterized in terms of stoichiometry and thickness. Compositional information about the near surface region is also important for the development of many other modern materials. Ion beam analysis techniques, such as Rutherford backscattering spectrometry (RBS) and elastic recoil detection (ERD) can provide this information in a uniform way for all chemical elements, including hydrogen. The film thickness is measured as atoms per area without relying on calibration standards. If the density of the film is known, its thickness in units of length can be deduced as well.

ERD is commonly performed with helium beams to measure hydrogen depth-profiles (Doyle and Peercy 1979). The technique can however be greatly extended when very heavy ion beams, such as gold, are used. With such heavy projectile beams ERD becomes sensitive to essentially all chemical elements. In contrast to RBS, ERD yields independent depth-profiles for every element. While the elemental resolution is best for the lighter elements $\mathrm{A}<50$, in many applications the heavier elements are also sufficiently resolved. In cases where the films contain heavier elements with similar atomic numbers, heavy ion ERD can be successfully augmented with heavy ion RBS. In contrast to standard RBS with helium beams, heavy ion RBS, e.g. oxygen or silicon

*Author for correspondence projectiles provides increased elemental and depth resolution. In addition, kinematic suppression (Ophel and Mitchell 1995) can be employed to reduce background from low- $z$ elements.

While heavy ion ERD and RBS have been used as analytical techniques previously, their versatility, efficacy, and sensitivity have recently been improved considerably through the use of large solid angle gas ionization detectors (Assmann et al 1994). In general, the use of gas ionization detectors overcomes the problem of radiation damage, which affects semiconductor detectors when used for the detection of heavy ions. Radiation damage leads to calibration instabilities and resolution deterioration. With regard to ERD analysis, gas detectors can easily be modified to allow both particle identification and energy measurement simultaneously. They can also provide directional information. This permits large solid angles for ERD and, thus, high sensitivity and reduced sample exposure, since the kinematic energy spread can be corrected.

This paper outlines the facility at the Australian National University for ion beam analysis with heavy projectile beams and gas ionization detectors (Timmers et al 1998; Ophel et al 1999). The efficacy and versatility of the techniques are demonstrated using several examples.

\section{Experimental}

Gas ionization detectors can provide relative energy resolutions of better than $1 \%$. Two basic designs have been employed for ion beam analysis. The axial design, 
often referred to as a Bragg-ionization chamber, has slightly better energy resolution, however, position information cannot readily be obtained with this set up. This can be achieved with the transversal design or parallel-plate detector. At the Australian National University a large solid angle, parallel-plate detector with position detection has been installed inside a large scattering chamber ( $2 \mathrm{~m}$ diameter) for ERD of heavy ions. This detector gives complete flexibility in the selection of the scattering geometry. A Bragg-ionization detector is currently being built for heavy ion RBS in the same vacuum chamber.

The design of the ERD system at the Australian National University is shown in figure 1. Following the impact of the Au projectile ions, at a beam energy of typically $200 \mathrm{MeV}$, recoil ions are ejected from the sample and enter the detector through a wire-supported mylar window $(0.5 \mu \mathrm{m}$ thick). The detector contains flowing propane gas maintained at a typical pressure of $8000 \mathrm{~Pa}$. Inside the detector, the recoil ions ionize the propane gas along their trajectory until they are fully stopped. The electrons and ions move in the electric field between anode and cathode and induce signals on the electrodes. The signal induced on the cathode depends on the vertical position of the ion track. A Frisch-grid at ground potential ensures that the signals induced on the second grid and the anode are independent of the vertical position. The combined anode signal and the signal from the second grid are proportional to the total energy $E$ of the recoil ion. The relative resolutions of these signals are of the order of $1 \%$ and $2 \%$, respectively. The anode is divided into four sections, labelled $\Delta E 1$, $\Delta E 2(\mathrm{R}), \Delta E 2(\mathrm{~L})$ and Residual. The $\Delta E 1$ and $\Delta E 2$ signals are proportional to the energy loss of the recoil ion in the gas volume underneath the respective electrode section. The energy loss $\Delta E$ is related to the atomic number of the ion and allows different chemical elements to be distinguished. The $\triangle E 2$ section is sub-divided into two segments right $(\mathrm{R})$ and left $(\mathrm{L})$ using a saw-tooth geometry. The horizontal position $x$ of the ion track can be obtained from the signals induced on these electrodes as:

$$
x=a \frac{R-L}{R+L},
$$

where $a$ is a calibration constant and $R$ and $L$ are the respective signal amplitudes. Likewise, the vertical position of the ion track is given by

$$
y=b \frac{\text { cathode }}{\Delta E 2+\text { residual }} .
$$

From $x$ and $y$ and the detector position the scattering angle $\theta$ can be calculated for every recoil ion. This information is necessary to correct the kinematic energy spread of the recoil ions over the solid angle $(5 \mathrm{msr})$. The corrected energy $E_{\text {cor }}$ is given by

$$
E_{\mathrm{cor}}=E\left(\frac{\cos \theta_{0}}{\cos \theta}\right)^{2},
$$

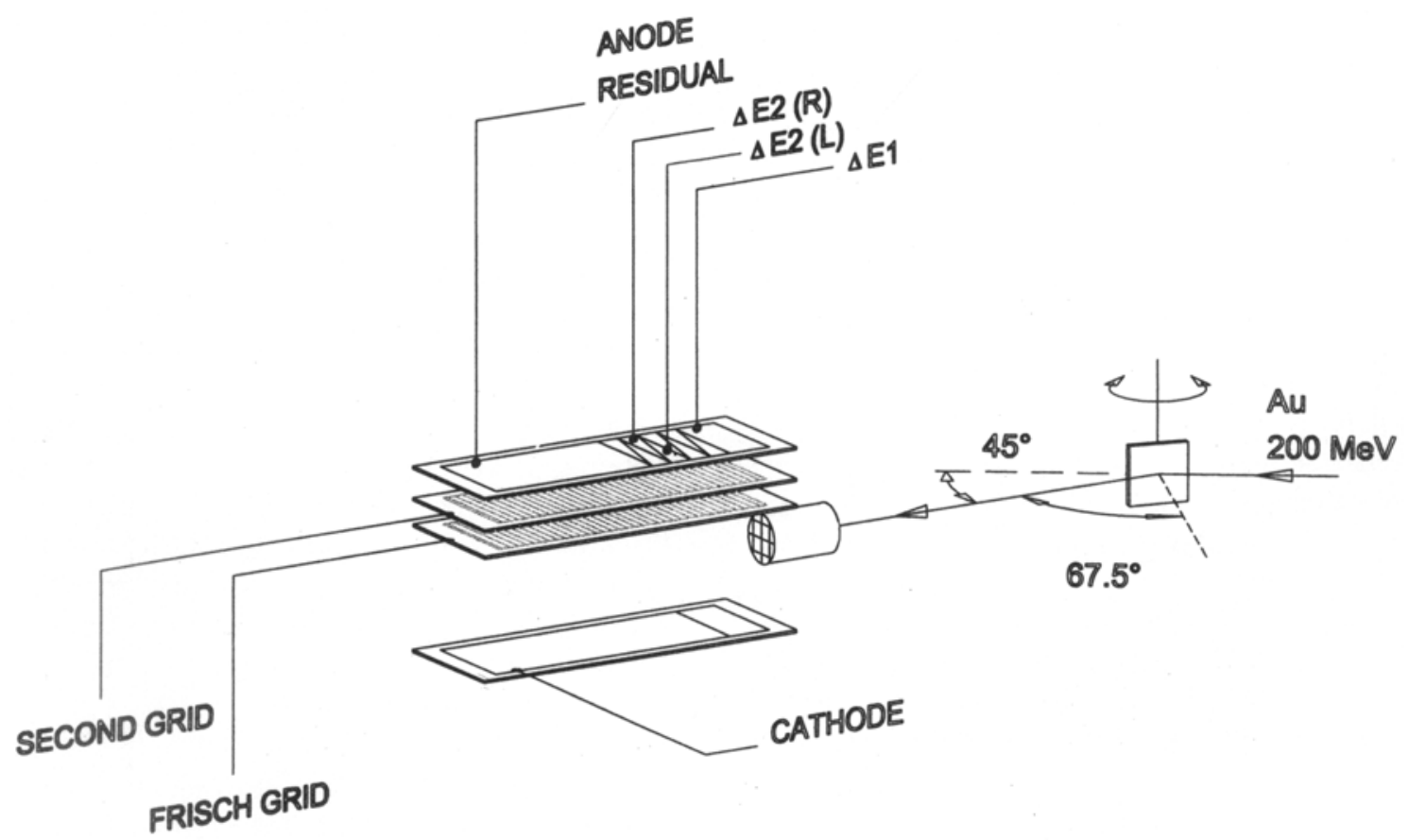

Figure 1. Schematic diagram of the ERD facility at the Australian National University. 
where $E$ is the detected energy and $\theta_{0}$ the scattering angle corresponding to the central detector axis. The ability to correct the kinematic energy spread allows large solid angles to be used whilst maintaining an energy resolution of $\sim 1 \%$.

Because of the energy loss of projectile and recoil ion inside the sample, the energy spectrum for a particular atomic number represents the depth-profile of the corresponding chemical element. This is illustrated in figure 2 for the elements $\mathrm{Ti}, \mathrm{Si}$ and $\mathrm{O}$ as measured for a multi-layer sample. The layer structure is reflected in the spectra, with the highest recoil energies corresponding to the sample surface.

\section{Characterization of Ge- and Sn-doped silica films for integrated photonics}

Photosensitive doped-silica films are being developed for applications in integrated photonics (Jarvis et al 1996). The photosensitivity of the films allows structures, such as wave guides or gratings, to be laser-imprinted. A series of silica films doped with $\mathrm{Ge}$ or $\mathrm{Sn}$ have been analyzed with the heavy ion ERD system at the Australian
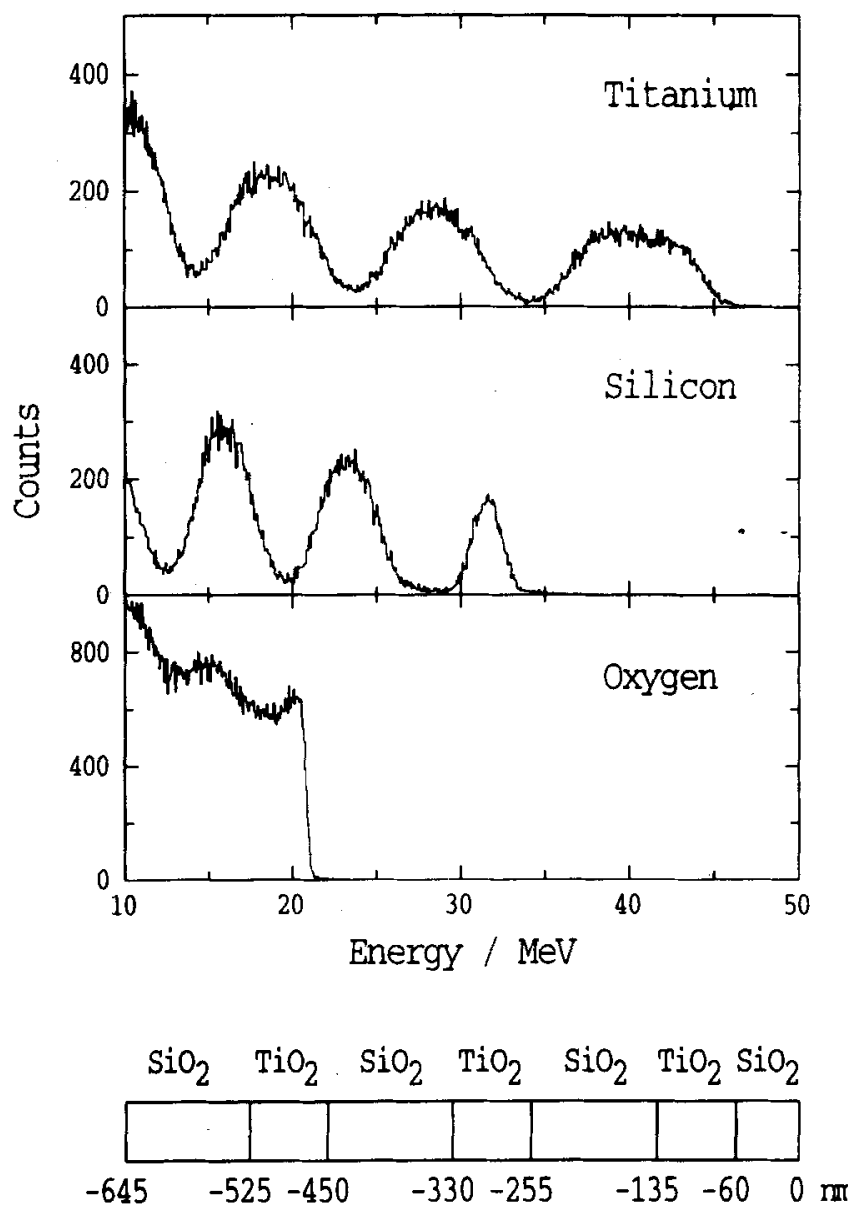

Figure 2. Energy spectra for $\mathrm{Ti}, \mathrm{Si}$ and $\mathrm{O}$ as measured with heavy ion ERD for the multi-layer sample illustrated at the bottom.
National University. A detailed account of these measurements is given elsewhere.

\section{Surface composition of high $T_{c}$ superconductors}

High $T_{\mathrm{c}}$ superconductors of the stoichiometry $\left(\mathrm{Bi}, \mathrm{Pb}_{2} \mathrm{Sr}_{2} \mathrm{Ca}_{2} \mathrm{Cu}_{3} \mathrm{O}_{10+x}\right.$ are being developed at the University of Wollongong, Australia (Rouessac 1998). The oxygen content and possible carbon contamination in the surface region of such samples affect their electrical properties. The sample stoichiometry and depth-profiles for $O$ and $C$ can be established with ERD of heavy ions. Figure 3 shows a two-dimensional plot of $\Delta E 1$ vs $E$. Both the $\mathrm{O}$ and $\mathrm{C}$ events are cleanly separated and can be projected out as energy spectra. However, Au scattering from the sample and $\mathrm{Bi}$ and $\mathrm{Pb}$ recoil ions obscure the events associated with $\mathrm{Ca}, \mathrm{Cu}$ and $\mathrm{Sr}$. This problem can be overcome by plotting other combinations of signals, such as $\Delta E 1$ vs $\triangle E 2$ and $\triangle E 2$ vs Residual. This is shown in figure 4 and 5 . Because of the different ranges of the ions in the detector and due to different dependencies of the stopping power on energy, $\mathrm{Ca}, \mathrm{Cu}$ and $\mathrm{Sr}$ and the integral of $\mathrm{Au}, \mathrm{Pb}$ and $\mathrm{Bi}$ can be isolated. This allows a complete and consistent set of energy spectra to be extracted for these samples. Using the code RUMP (Doolittle 1985), the sample stoichiometry was simulated. The simulation agrees with the data when the nominal stochiometry is assumed with oxygen as $\mathrm{O}_{13}$. The $O$ energy spectrum for a sample annealed in air is shown in figure 6 in comparison with the theoretical simulation. It can be seen that a reduction of the $O$ concentration occurs in the surface region over the first $400 \mathrm{~nm}$. The spectra for the other chemical elements do not show this feature, so that surface roughness effects which can cause a similar fall-off are unlikely.

\section{Depth-profiles of magnetic films for hard discs}

Magneto-resistive hard discs for data storage are well suited for analysis by heavy ion ERD. These discs consist of thin surface films deposited on typically a NiP substrate. The magneto-resistive layer $(\mathrm{CoTaCr})$ is protected by a hard coating of hydrogenated carbon or CNH. As part of a benchmark study (Mitchell 1998), which attempted to evaluate the efficacy of different ion beam analysis techniques for this application, several such hard disc materials were analyzed with the heavy ion ERD system at the Australian National University.

Figure 7 shows a typical $\Delta E 1$ vs $E$ plot. The sample structure is already obvious from this plot, when the energy scale is translated into the depth-scale. On top of the NiP substrate are an elemental $\mathrm{Cr}$ layer and a layer comprising $\mathrm{Cr}$, $\mathrm{Co}$ and $\mathrm{Ta}$. The tantalum events are not evident in this particular plot, because the $\Delta E 1$ signal is greater than 200 channels (ch), but they can 
be extracted using the $\triangle E 2$ signal. The magnetic layer is covered by a thin hydrogenated $\mathrm{C}$ protective coating. $O$ occurs only at the very surface and is most likely due to sample handling in air. After extracting the individual energy spectra for all elements, the stoichiometry and thickness of every layer can be determined. The data from ERD is currently being compared with results from other ion beam analysis techniques.

\section{Detection of heavy elements with kinematic supression}

Elements heavier than $A \sim 50$ are sometimes more easily resolved with RBS than with heavy ion ERD. A problem arises when traces of heavy elements are implanted into matrices consisting of light elements, such as silicon or silica. The RBS signal from the implanted atoms can

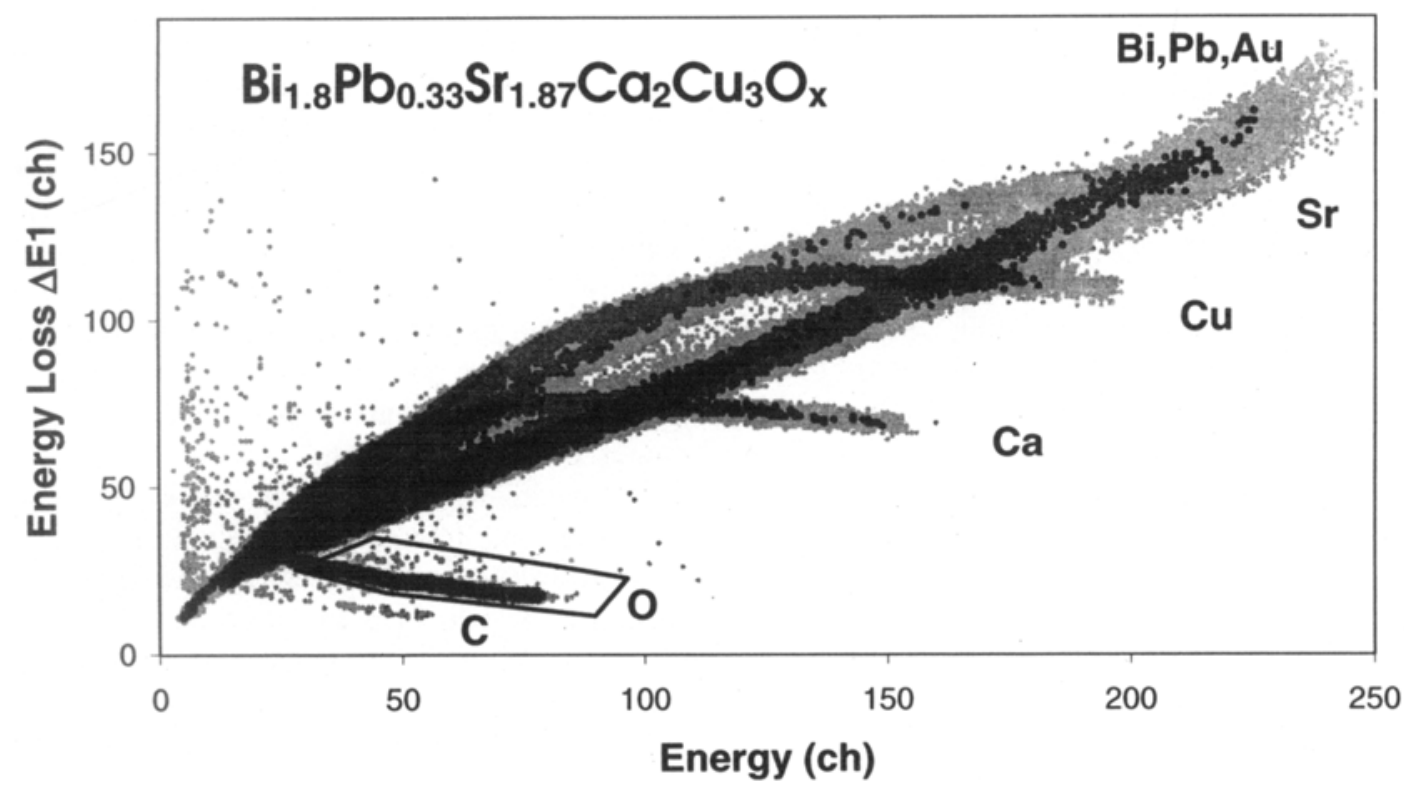

Figure 3. Two-dimensional plot of $\Delta E 1$ vs $E$ for a high $T_{\mathrm{c}}$ superconductor (The stoichiometry is given in the figure). A typical window to select the $O$ recoils is shown.

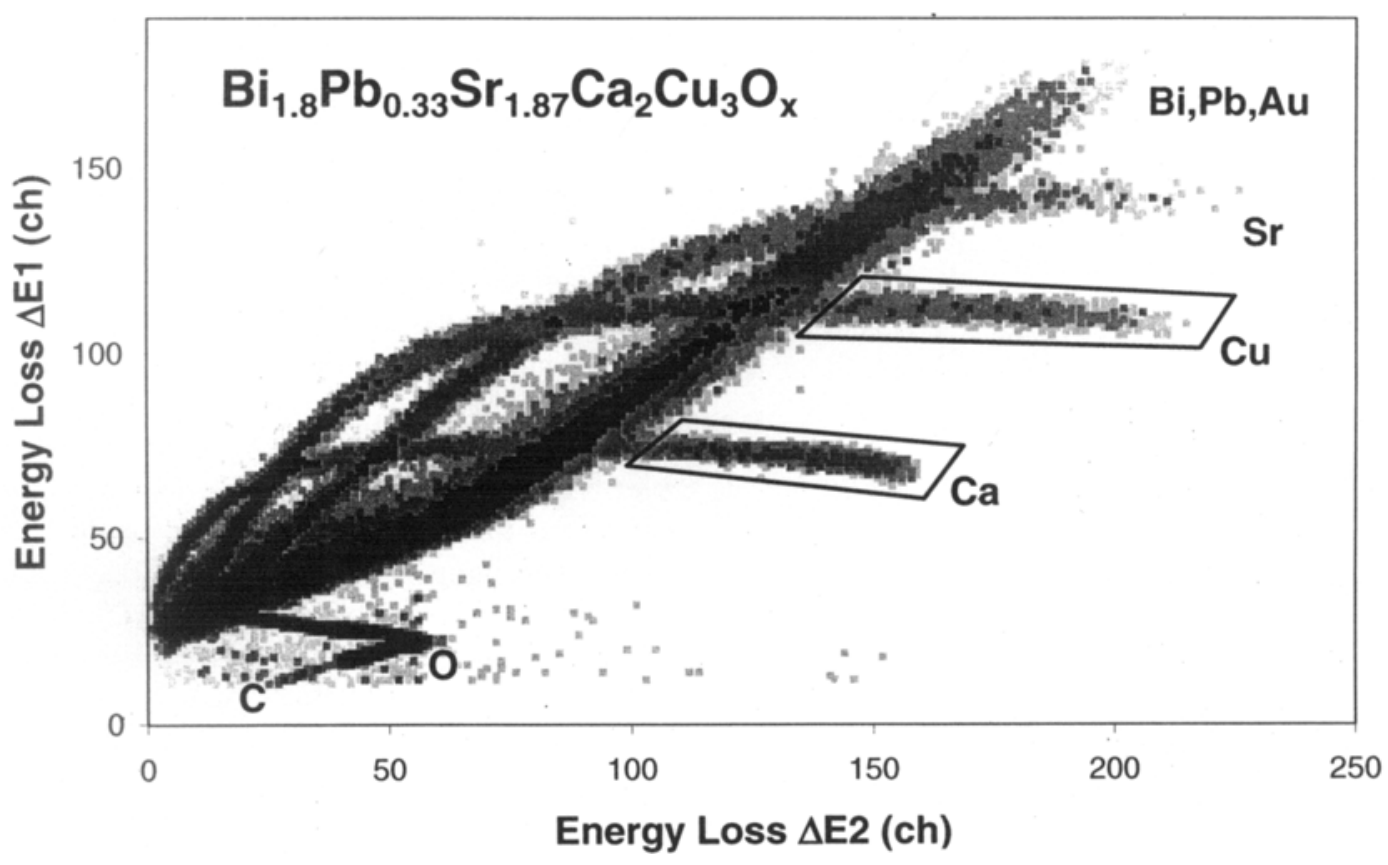

Figure 4. Two-dimensional plot of $\Delta E 1$ vs $\Delta E 2$ for the same high $T_{\mathrm{c}}$ superconductor. Typical windows to select the Ca and $\mathrm{Cu}$ recoils are shown. 
then be obscured by the strong background from the matrix. This problem can be overcome when projectile beams such as $\mathrm{Si}$ or even heavier ions are used for the RBS measurement. The scattering kinematics then excludes scattering of the projectile ion from the lighter matrix atoms to scattering angles backward of $90^{\circ}$. This is often referred to as kinematic uppression. This way clean depth-profiles can be obtained for the heavy trace elements which are not kinematically suppressed. This has been demonstrated for Au implanted into silicon at $90 \mathrm{keV}$ and subsequent annealing of the sample. Using an $18 \mathrm{MeV} \mathrm{Si}$ beam, Si scattered of $\mathrm{Au}$ was observed at a scattering angle of $100^{\circ}$ using a silicon surface barrier detector (Ophel and Mitchell 1995). The spectrum

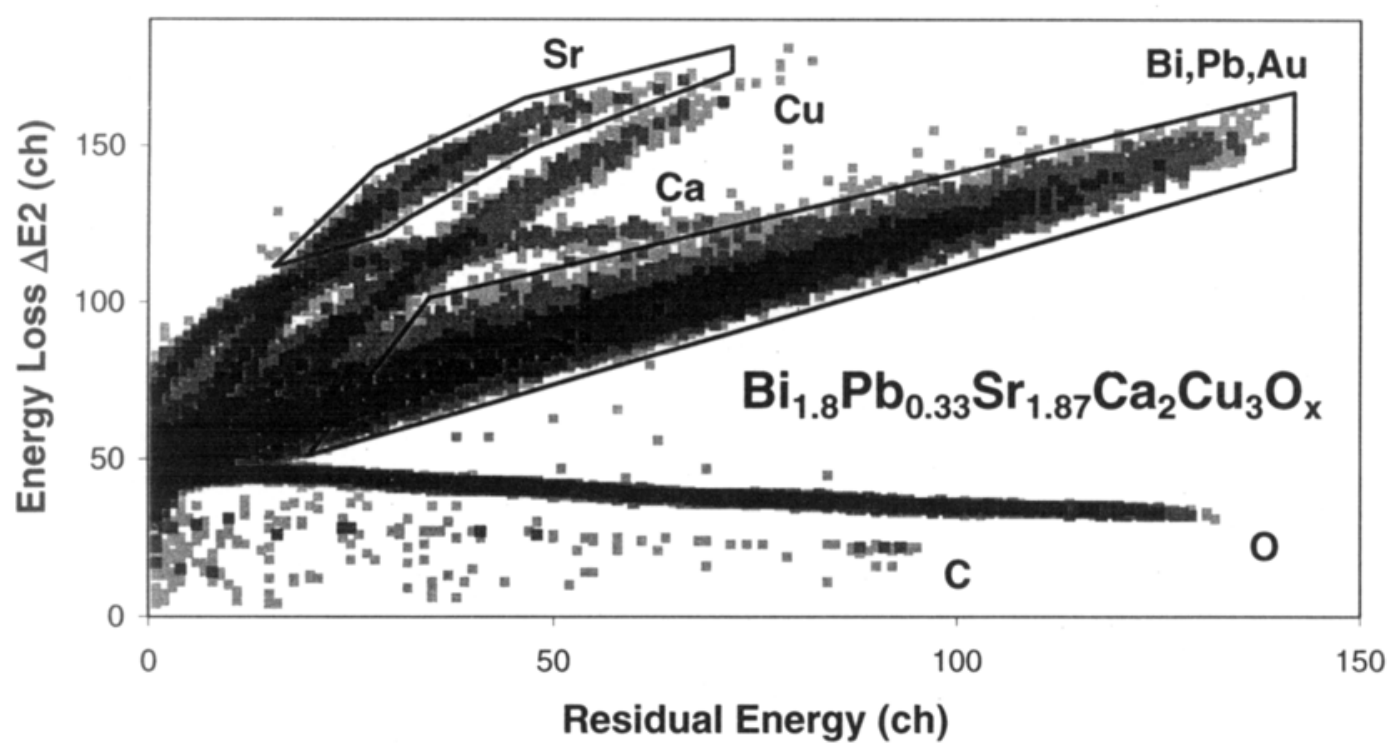

Figure 5. Two-dimensional plot of $\Delta E 2$ vs residual energy for the same high $T_{\mathrm{c}}$ superconductor. Typical windows to select the $\mathrm{Sr}$ recoils and the integral of the $\mathrm{Au}, \mathrm{Bi}$ and $\mathrm{Pb}$ ions are shown.

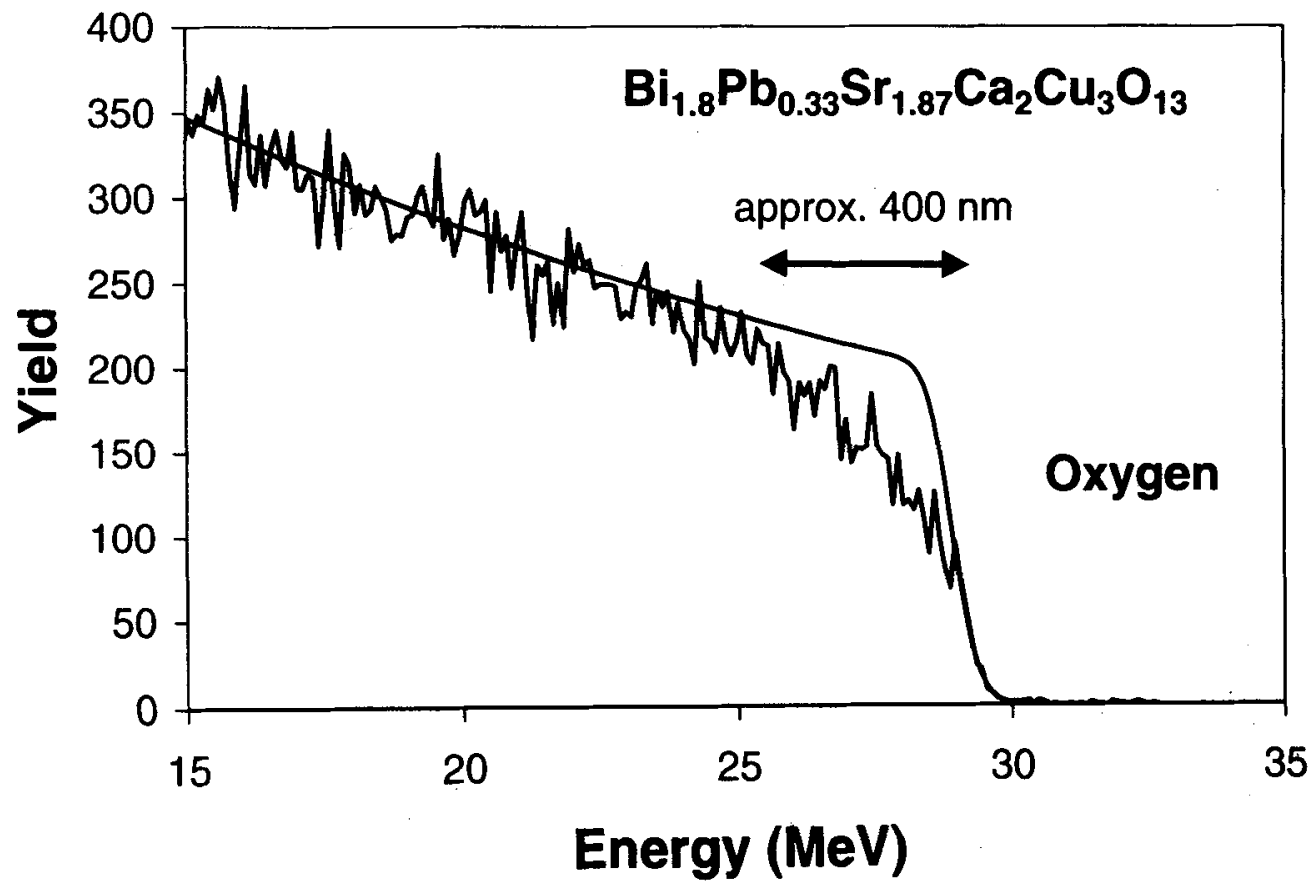

Figure 6. Energy spectrum for $O$ as measured for a high $T_{c}$ superconductor annealed in air in comparison with a simulated spectrum assuming a uniform stoichiometry of $\mathrm{O}_{13}$. 


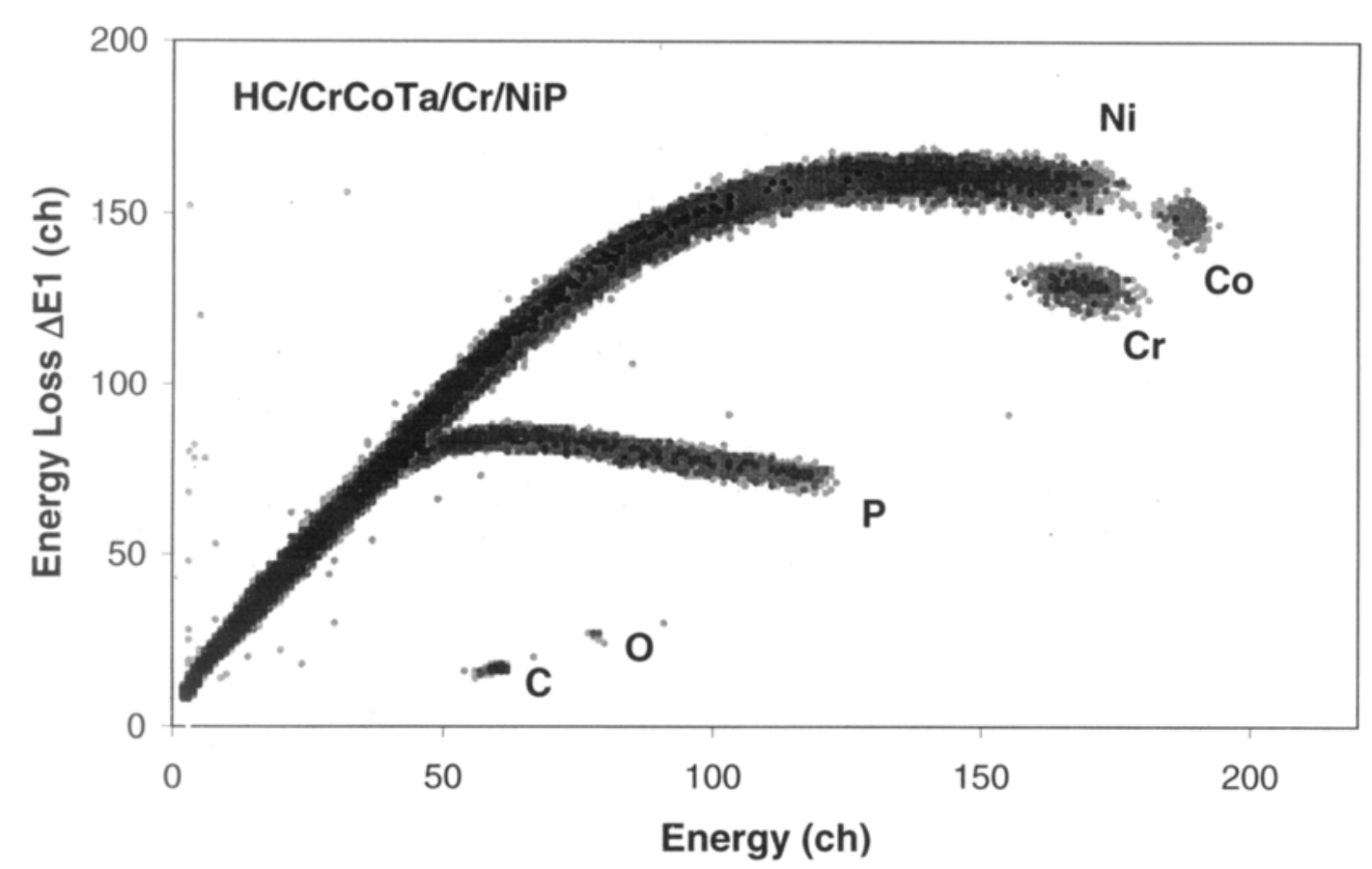

Figure 7. Two-dimensional plot of $\Delta E 1$ vs $E$ for the surface of a magnetic hard-disk.

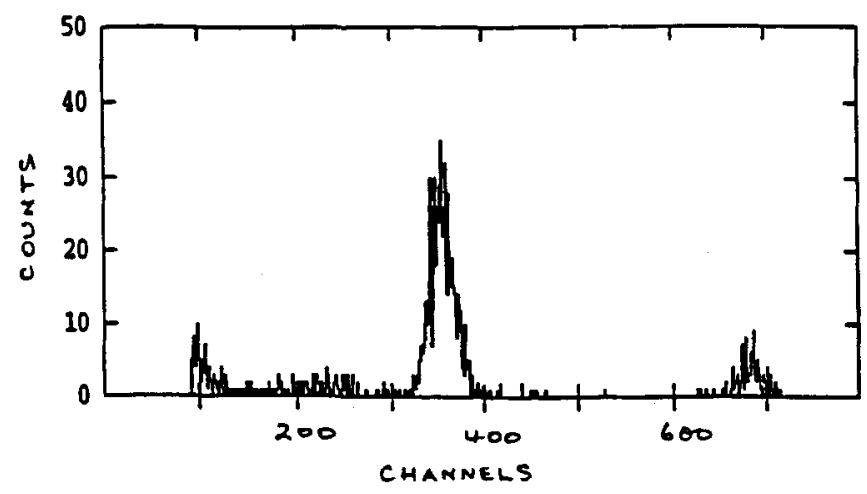

Figure 8. Heavy ion RBS energy spectrum (not calibrated) of Si ions scattered to $100^{\circ}$ from Au implanted in Si. Scattering from the Si matrix is kinematically suppressed.

is shown in figure 8. In addition to the strong peak at a depth of $1 \mu \mathrm{m}$ which corresponds to Au diffused into a void region during annealing, residual $\mathrm{Au}$ at the surface and possibly untrapped $\mathrm{Au}$ beyond the void region are apparent.

\section{Conclusions}

The use of heavy ion projectile beams expands the versatility of ion beam analysis techniques such as Rutherford backscattering spectromety (RBS) and elastic recoil detection (ERD). Heavy ion ERD is sensitive to all chemical elements and absolute depth-profiles in atoms per unit area can be obtained without the need for measurements of standards. The use of gas ionization detectors increases detection efficiency and minimizes the beam exposure of the samples, so that the analysis is essentially nondestructive. The sampling depth of a few micrometers makes these techniques ideal for the stoichiometric analysis of the surface region of homogeneous materials and, in particular, thin surface films.

\section{Acknowledgements}

The authors would like to thank Dr Vincent Rouessac for making available samples of high $T_{\mathrm{c}}$ superconductors fabricated by him. They are also grateful to Prof. Ian Mitchell and Ms Tessica Weijers for their contributions to this work.

\section{References}

Assmann W et al 1994 Nucl. Instrum. Meth. $\mathbf{B 8 5} 726$

Doolittle L R 1985 Nucl. Instrum. Meth. B9 344

Doyle B L and Peercy P S 1979 Appl. Phys. Lett. 34811

Jarvis R A et al 1996 Electron. Lett. 32550

Mitchell I V 1998 (private communication)

Ophel T R and Mitchell I V 1995 Proceedings of the Australian conference on nuclear techniques of analysis (Sydney: Australian Institute of Nuclear Science and Engineering) p. 80

Ophel T R, Timmers H and Elliman R G 1999 Nucl. Instrum. Meth. A423 381

Rouessac V 1998 (private communication)

Timmers H et al 1998 Nucl. Instrum. Meth. B136-138 611 